\title{
ICD-11 should not repeat the mistakes made by DSM-5
}

\author{
Allen J. Frances and John M. Nardo
}

\section{Summary}

Having two systems of psychiatric diagnosis creates unnecessary confusion therefore it would be desirable to achieve increased consistency between ICD-11 and DSM-5. Unfortunately, however, DSM-5 has included many controversial suggestions that have weak scientific support and insufficient risk-benefit analysis. As a result ICD-11 should learn from the DSM-5 mistakes rather than repeating them.

\section{Declaration of interest}

None.
Allen J. Frances (pictured) was chair of the DSM-IV Task Force and of the Department of Psychiatry at Duke University School of Medicine. He is currently professor emeritus at Duke University. John Nardo is a retired psychiatrist, formerly at the Department of Psychiatry and Behavioral Sciences at Emory University. Both have actively followed DSM-5 development on their respective blogs.

\section{Background}

The ICD and DSM diverged only by unfortunate historical accident, not necessity. The differences are relatively trivial, yet big enough to cause confusion. Neither can claim superiority just as no language has priority over its near neighbours. In the best of worlds, there would not be two different diagnostic languages to create a Babel of divided loyalty among psychiatrists.

Twenty-five years ago, those of us who were preparing DSM-IV ${ }^{1}$ and ICD- $10^{2}$ worked together to bring them towards closer alignment. Although succeeding in eliminating some of the more foolish differences, we failed to achieve anything like real unification. The remaining differences reflected stubbornly held opinions of the experts on both teams; the differing institutional priorities of their sponsors (the American Psychiatric Association (APA) and the World Health Organization (WHO)); and perhaps a dose of shared sibling rivalry.

The simultaneous preparation of DSM- $5^{3}$ and ICD-11 provided another, perhaps even more favourable opportunity to achieve increased compatibility. Whereas DSM-IV and ICD-10 both offered competing criteria sets, this time round ICD-11 has chosen against rigid criteria, instead offering more flexible, prototypal descriptions. This opened up the possibility for a completely complementary and nested, rather than competing, relationship. There could have been an agreed equivalence of each diagnostic construct, expressed in two formats suitable for different settings and purposes. The briefer, simpler ICD-11 descriptions could be used when time and practical clinical convenience was the priority. The more structured DSM-5 criteria sets would be the standard for research, forensics and those clinical settings that allow for more precise and reliable diagnosis. However, such cooperative development of compatible modular systems did not occur. The groups working on ICD-11 and DSM-5 held some joint meetings and agreed on a revision of the overall table of organisation, but the definitions of the disorders and which should be included have not been coordinated.

The date for publishing ICD-11 was originally meant to be $2011 / 2012$, but delays have postponed it until 2015/2016 at the earliest. This creates a decision point for ICD-11. With the final version of DSM-5 now available, the ICD-11 groups have a choice to either aim for increased compatibility with DSM-5, or make their own independent decisions. Normally, compatibility would be a high priority. Most of the variations between DSM-IV and ICD-10 reflected only the meaningless narcissism of small differences - creating trouble without adding value. But these are not normal circumstances; DSM-5 has made many controversial decisions that are scientifically unsupported and clinically unsafe ${ }^{4}$ and ICD-11 would be well advised not to follow its lead.

\section{Problems with DSM-5}

\section{Sensitivity v. specificity}

The consistent bias in DSM-5 has been to increase the sensitivity of psychiatric diagnosis and to reduce its specificity. The scope of diagnosis has expanded by both loosening the thresholds for existing diagnoses and introducing new disorders at the fuzzy, elastic and populous boundary with normality. This diagnostic imperialism was a natural consequence of the instructions given to the experts working on DSM-5. They were encouraged to be innovative and paradigm shifting - anything and everything was 'on the table."

Experts always worry more about false negatives than false positives, and given freedom, tend to expand their area of interest. Seemingly small changes in each section can have an enormous cumulative impact on the rates of misdiagnosis and on the prescription of unneeded and potentially harmful treatment. It seems that DSM-5 looks to turn the existing diagnostic inflation in psychiatry into reckless hyperinflation. ${ }^{6}$ It has included a number of new and untested mental disorders without sufficient data on prevalence, reliability, validity, treatment response, and risk/benefit ratio. The results of DSM-5 field testing for reliability were abysmally low by historical standards. ${ }^{7,8}$

The new diagnoses share being understudied and overinclusive. 'Somatic symptom disorder' will mislabel many of the medically ill as also mentally ill. 'Disruptive mood dysregulation disorder' can turn temper tantrums into mental disorder. 'Minor neurocognitive disorder' will have a high false-positive rate among those with the everyday forgetting of old age. Binge eating disorder requires only one binge a week for 3 months.

In addition, DSM-5 has lowered the thresholds of existing diagnoses - making it far too easy to diagnose major depressive disorder in people experiencing normal grief; ${ }^{9}$ attention-deficit disorder in adults eager to get stimulant drugs for performance 
enhancement and recreation; substance addiction in youngsters who have only occasional harmful substance use; and posttraumatic stress disorder in legal and disability settings.

This edition has also created a slippery slope by introducing the concept of 'behavioural addictions'- with gambling as its first example. This premature precedent can eventually spread to make a mental disorder of any passionate interest - opening the door to the fad diagnosis of internet, sex, shopping and other untested 'addictions'.

\section{Personality disorder}

In DSM-5 the task of creating a needed dimensional system for diagnosing personality disorders was undertaken. But its product was a hybrid monster, way too complicated for clinical practice. Dimensional diagnosis of personality disorders went so far off track that it had to be dropped at the last minute - leaving DSM-5 stuck with the same inadequate categorical method that informed DSM-IV and ICD-10. What is desperately needed is a simple dimensional system, one that can be easily understood and readily used by the average clinician.

\section{Major depressive disorder}

However, DSM-5 did not take a serious look in the other direction - the possibility of tightening diagnoses that badly need tightening. The best example is major depressive disorder. This was created by DSM-III ${ }^{10}$ by collapsing a number of formerly separate diagnoses as divergent as 'melancholia' and 'depressive neurosis' into one category. The resulting heterogeneity has complicated clinical trials, basic genetic and other research, treatment approaches and efforts to curb overmedication. ${ }^{11}$ Mild major depressive disorder is a contradiction in terms - often not 'major', not 'depressive', and not 'disorder'. Instead of removing the 'bereavement exclusion', DSM-5 should have considered extending a similar exclusion for sadness and other mild 'depressive-like' symptoms in the face of other severe life stress such as divorce, job loss or financial troubles. ${ }^{12}$

\section{Anxiety disorders}

At one point, DSM-5 considered even lowering the threshold of generalised anxiety disorder and adding mixed anxiety depression. Fortunately, both of these suggestions were squashed, but they failed to consider tightening the generalised anxiety disorder criteria set, particularly when an individual faces a realistically stressful situation. The current boundary with normal worry is far too porous, allowing the excessive use of potentially dangerous anxiolytic medicine. Similarly, there needs to be a strengthening of the boundary between everyday normal fears and the mental disorders, social anxiety and simple phobia.

\section{The challenge facing ICD-11}

Given DSM-5 has made so many questionable decisions ICD-11 would be well advised not to follow its lead. But this will be no easy task. There is an implicit authority to the written word and the published page, creating strong pressures on the ICD-11 workers to be led astray by DSM- 5 precedents. The DSM- 5 changes, however poorly conceived, will have gained a kind of tenure and the apparent authenticity of having been accepted after a laborious decision process. There will be direct appeals to harmony. And a number of DSM-5 veterans are also working on ICD-11. However, following DSM-5 as a template would be a serious mistake. The DSM-5 decisions were based on a secretive and closed process that minimised risks while overvaluing hypothetical benefits. The field trials failed to measure the impact on diagnostic rates and produced reliabilities falling far below historical standards - often falling back to the terrible lack of reliability that preceded DSM-III. Also, when all its production deadlines were missed, DSM-5 cancelled its badly needed quality control step. The DSM-5 has been hurried to press prematurely without proper testing.

The new edition of ICD can and must do better - learning from the DSM-5's mistakes rather than perpetuating them. It must set itself a higher standard of scientific evidence before changes are made, ensure a greater openness of process and be more responsive to differing viewpoints. Compared with APA financial profligacy in producing DSM-5, the WHO budget for ICD-11 is remarkably thin, but wisdom and caution can easily trump availability of resources when it comes to producing a safe, enduring diagnostic system.

Allen J. Frances, MD, Duke University, Durham, North Carolina; John M. Nardo MD, formerly Department of Psychiatry and Behavioral Sciences, Emory University, Atlanta, Georgia

Correspondence: John M. Nardo, MD. Email: jnardo@ellijay.com

First received 7 Feb 2013, accepted 13 May 2013

\section{References}

1 American Psychiatric Association. Diagnostic and Statistical Manual of Mental Disorder (4th edn) (DSM-IV). APA, 1994.

2 World Health Organization. The ICD-10 Classification of Mental and Behavioural Disorders: Clinical Descriptions and Diagnostic Guidelines. WHO, 1992.

3 American Psychiatric Association. Diagnostic and Statistical Manual of Mental Disorder (5th edn) (DSM-5). APA, 2013

4 Frances A. Whither DSM-V? Br J Psychiatry 2009; 195: 391-2.

5 Frances A. The first draft of DSM-V. BMJ 2010; 340: C1168.

6 Batstra L, Frances A. Diagnostic inflation: causes and a suggested cure. J Nerv Ment Dis 2012; 200: 474-9.

7 Kraemer HC, Kupfer DJ, Clarke DE, Narrow WE, Regier DA. DSM-5: how reliable is reliable enough? Am J Psychiatry 2012; 169: 13-5.

8 Regier DA, Narrow WE, Clarke DE, Kraemer HC, Kuramoto SJ, Kuhl EA, et al. DSM-5 field trials in the United States and Canada, Part II: test-retest reliability of selected categorical diagnoses. Am J Psychiatry 2013; 170: 59-70.

9 The Lancet. Living with grief. Lancet 2012; 379: 589.

10 American Psychiatric Association. Diagnostic and Statistical Manual of Mental Disorder (3rd edn) (DSM-IIII). APA, 1980.

11 Parker G, Fink M, Shorter E, Taylor MA, Aiskal H, Berrios G, et al. Issues for DSM-5: whither melancholia? The case for its classification as a distinct mood disorder. Am J Psychiatry 2010; 167: 7.

12 Friedman RA. Grief, depression, and the DSM-5. N Engl J Med 2012; 366: 1855-7. 